\title{
Fluoruros tópicos : Revisión sobre su toxicidad
}

Barbería E, Cárdenas D, Suárez M, Maroto M. Fluoruros tópicos: Revisión sobre su toxicidad. Rev Estomatol Herediana 2005;15(1): 86 - 92

\section{RESUMEN}

Los fluoruros se han utilizado durante décadas para prevenir la caries y su eficacia está aceptada aunque no así su vía de administración. Actualmente se preconiza la utilización tópica de los compuestos de fluoruro para maximizar sus beneficios y minimizar sus riesgos. La utilización racional de la vía tópica entraña la educación de la población y un cambio en los profesionales de la salud para asumir que preparados como las pastas dentales y los colutorios que se consideran productos cosméticos son medicamentos con riesgos ciertos si no se utilizan adecuadamente. La concentración excesiva del preparado activo y la ingesta sistemática de pequeñas cantidades pueden desarrollar fluorosis si ocurre cuando el niño no ha completado la formación de sus coronas dentarias. Asimismo, la ingestión de cantidades importantes pero posibles puede dar lugar a un accidente agudo de carácter grave. Los médicos pediatras y en general los profesionales de la salud deben conocer los beneficios y riesgos de estos preparados, determinar la ingesta total de flúor de cada uno de sus pacientes y prescribir los que están indicados. Los padres tienen que comprender que los preparados tópicos a base de fluoruro son medicamentos y deben ser manejados como tales siendo ellos responsables de que no ocurran accidentes por mal uso. Los fabricantes deberían modificar sus estrategias comerciales y evitar envases de contenido excesivo así como incluir información detallada sobre sus componentes, tanto del principio activo como del contenido de alcohol.

\section{Palabras clave: FLUORUROS TÓPICOS. toxicidad}

\section{Topic fluorides: a revision on its toxicity}

\section{ABSTRACT}

Fluorides have been used for decades to prevent dental caries, and their efficacy is accepted although not the delivery method. Currently it praises the topical use of fluoride compounds to maximize benefits and minimize risks. Rational topical use entails education of the population and health professionals' so that they recognize that preparations like toothpastes and mouth rinses, some of them considered cosmetic products, are indeed medicines with real risks involved if they are not used properly. Excessive concentration of the active component and frequent ingestion of small amounts of fluoride during tooth development may cause dental fluorosis. Furthermore, accidental ingestion in great amounts can cause an acute intoxication. Paediatrician and in general health professionals should know the benefits and risks of these substances, and determine the total fluoride intake of each of their patients in order to prescribe what is indicated. Parents have to understand that topical fluoride preparations are medications and should be handled as such, assuming the responsibility of preventing accidents due to misuse. Manufacturers should modify their commercial strategies, avoiding packaging with excessive content and also including detailed information of the product, not only of the active component but also of the alcohol content.

Keywords: TOPICAL FLUORIDES. toxicity
Elena Barbería Leache ${ }^{1}$ Dora Cárdenas Campos ${ }^{2}$ María Cruz Suárez Clúa ${ }^{3}$ Myriam Maroto Edo $^{3}$

Catedrática del Departamento de Profilaxis, Odontopediatría y Ortodoncia.

2Alumna del Máster en Odontopediatría. ${ }^{3}$ Profesora Asociada del Departamento de Profilaxis, Odontopediatría y Ortodoncia.

Facultad de Odontología. Universidad Facultad de Odonto

\section{Correspondencia}

Elena Barbería Leache,

Facultad de Odontología - UCM

Plaza de Ramón y Cajal s/n, 28040

Madrid España.

Telef: (0034) 913942044

E-mail: barberia@odon.ucm.es

Aceptado para publicación : 29 de junio del 2005

\section{Introducción}

Los mecanismos propugnados para la prevención de la caries dental son múltiples y algunos de ellos bien conocidos. La eliminación de las colonias bacterianas utilizando métodos de arrastre como son el cepillo y la seda dental, así como disminuir el consumo de hidratos de carbono, han demostrado gran efectividad. Sin embargo, se considera que la creciente exposición a los fluoruros en diversas formas y vehículos tiene un peso decisivo en la disminución de la prevalencia de caries dental observada en las últimas décadas en los países industrializados (1-8).

Los agentes fluorados de uso tópico son ampliamente comercializados y están a disposición del público en supermercados o parafarmacias ya que, algunos de ellos, se acogen a la legislación de los cosméticos. Consideramos que esto puede ser peligroso ya que como fármacos que son no están exentos de contraindicaciones y riesgos.

El propósito del presente artículo es revisar las consideraciones y proporcionar recomendaciones en relación a la utilización de los fluoruros tópicos, para maximizar sus beneficios y prevenir su toxicidad.

\section{Fluoruros}

Se sabe que, tras la formación de las coronas dentarias y antes de la emergencia del diente, el fluoruro presente en los fluidos que contactan con la corona comienza a incorporarse a los tejidos mineralizados sustituyendo a grupos hidroxilo y formando hidroxiapatita. La cantidad de flúor incorporado es mayor en la zona externa del esmalte que en el interior y mayor también en los dientes permanentes que en los tempo- rales si hacemos la medición en el momento de la emergencia dentaria $(9,10)$.

Tras la erupción del diente, durante la maduración posteruptiva, se incorporan iones fluoruro procedentes de la saliva. Si la concentración de iones flúor en la saliva y en la placa dental es elevado se formarán cristales de fluorapatita con mayor resistencia a la desmineralización (9,11-14).

Las propiedades preventivas del ión fluoruro se atribuyen a tres mecanismos de acción $(10,15)$ :

1. Favorece la remineralización, incorporándose a los nuevos cristales de fluorapatita y dando, como consecuencia, una superficie más resistente. Aunque todavía es motivo de estudio, la aplicación frecuente de dosis bajas de fluoruro de forma tópica sobre lesiones de mancha blanca y en presencia de iones de 
calcio y fosfato favorece una remineralización más profunda que si las dosis de fluoruros aplicadas tópicamente fuesen más altas. Podría entenderse como si las concentraciones elevadas dieran lugar a una capa superficial muy remineralizada y poco porosa que impediría el paso de iones a zonas más profundas (16).

2. Inhibe la desmineralización. Los iones fluoruro penetran a la estructura dentaria simultáneamente con la pérdida de minerales durante el ataque ácido. En este sentido se ha comprobado que el fluoruro presente es mucho más efectivo que el fluoruro incorporado al esmalte durante su formación. Por tanto, es necesario el flúor tópico para proteger de la desmineralización (17).

3. Inhibición de la actividad bacteriana. El ión flúor tiene acción sobre el crecimiento de la placa, como agente bactericida $(18,19)$. Su mecanismo de acción es múltiple. Disminuye la capacidad de entrada de carbohidratos a las bacterias y por tanto disminuye la formación de ácidos. Asimismo interfiere con la biosíntesis de los polisacáridos extracelulares disminuyendo la adhesión al esmalte.

Actualmente la acción preventiva de los fluoruros frente a la caries no se discute, sin embargo su utilización debe ser cuidadosa para lograr su efecto beneficioso limitando sus efectos tóxicos. El conocimiento, aunque sea somero, de su metabolismo y capacidad tóxica nos permitirá evitar correr riesgos innecesarios.

\section{Metabolismo del fluor}

El aporte de flúor al organismo es por vía oral en su mayor parte. Muchos alimentos lo contienen en mayor o menor medida. Unas veces está presente de forma natural como en el pescado o en el té y otras veces es agregado en el proceso de elaboración y manufactura como ocurre en algunos alimentos infantiles, sal de cocina, leche y otros. Mención especial merece el flúor presente en las aguas de consumo. La cantidad es muy variable. Puede ser excesiva (superior a $1 \mathrm{ppm}$ ), inexistente o estar presente en cualquier concentración en este rango. La procedencia puede ser natural (aguas de zonas con minerales ricos en flúor) o ser añadida artificialmente (20-24).

El uso racional de los fluoruros entraña el conocimiento previo del aporte total de flúor de una persona. Se hace necesario conocer la concentración en el agua de la zona en que vive, las bases de su alimentación, y si incluye rutinariamente elementos fluorados (25-27).

El flúor procedente del agua se absorbe prácticamente en su totalidad mientras que el flúor que procede de los alimentos se absorbe entre $50-80 \%$. El flúor procedente de la inhalación es esporádico y no representa una cantidad significativa. La absorción mayor ocurre en el estómago y en menor medida en el intestino. A los 30 minutos de la ingesta, el $40 \%$ ya se encuentra en los líquidos circulantes. A las cuatro horas el $90 \%$ ya se ha absorbido desde el tubo digestivo. La excreción se realiza fundamentalmente por el riñón (60-70\%), heces (5-10\%) y en pequeñas cantidades por otras secreciones corporales (28-30).

El nivel de fluoruros en saliva es aproximadamente $0,01 \mathrm{ppm}$, pero pueden existir variaciones. Algunos estudios muestran que un cambio en la concentración de fluoruro salivar de 0,01 ppm a 0,02 ppm puede ser suficiente para que un niño sea caries-activo o cariesresistente. La placa dental es también un reservorio de fluoruro. Aquí se encuentra en proporciones más altas que en la saliva (14-20 ppm). La mayor parte se encuentra combinado con el calcio pero puede liberarse cuando el $\mathrm{pH}$ desciende (31).

\section{Toxicidad de los fluoruros}

Los riesgos de la utilización de fluoruros se derivan de una ingesta excesiva sea a corto o a largo plazo.

La toxicidad aguda es un cuadro grave que resulta de la ingestión de grandes dosis de fluoruros. Si la cantidad es suficiente puede acusar con la muerte del niño. Esto no es solamente una posibilidad sino que numerosos trabajos publicados por los Servicios de Salud de diversos países, muestran que la hospitalización de pacientes infantiles por consumo de dosis tóxicas de fluoruros es relativamente frecuente $(32,33)$.

Se considera que la dosis tóxica probable (DTP) es de $5 \mathrm{mg} / \mathrm{kg}$ de peso cor- poral. En el caso de ingesta masiva de flúor el riesgo de accidente agudo dependerá, por tanto, del peso del niño. Los accidentes graves y con riesgo de toxicidad aguda suelen provenir de la ingestión masiva de suplementos de flúor administrado en forma de pastillas. Sin embargo, hay que considerar la posibilidad de que una dosis excesiva provenga de otras fuentes $(32,33)$.

La toxicidad crónica se deriva de la ingestión continua de pequeñas dosis de fluoruros pero que son suficientes, por su efecto acumulativo, para provocar la fluorosis dental (1,9,34-38). Los mecanismos por los que se produce la fluorosis no son todavía bien conocidos. Se le atribuye una acción tóxica sobre los ameloblastos, disminuyendo su número e interfiriendo en la maduración y mineralización del esmalte maduro $(9,39)$.

Los efectos de la ingestión de fluoruros parecen ser acumulativos durante la etapa formativa del diente. La duración de la exposición a los fluoruros durante la amelogénesis será el determinante más importante al explicar el desarrollo de fluorosis dental $(1,38,40,41)$. Se considera que el riesgo es menor durante la fase secretora (antes de los quince meses de edad) y mayor si se administra durante el estadio de maduración del esmalte $(9,38,39)$.

Los primeros años de vida son los más críticos para el desarrollo de fluorosis en los incisivos centrales permanentes, los de principal implicación estética, reportándose el mayor riesgo entre los 21 y 30 meses de edad para las niñas y 15 y 24 meses para los niños. Los dientes que se desarrollan y mineralizan más tarde, como los premolares, tienen una mayor prevalencia de fluorosis y son afectados más severamente. Los dientes primarios también presentan riesgo de desarrollo de fluorosis, en particular los segundos molares primarios $(42,43)$.

La fluorosis puede presentar grados. La manifestación menor son manchas blancas opacas con una zona superficial mineralizada y una zona subsuperficial hipocalcificada que le da un aspecto opaco y una mayor fragilidad. Cuando aumenta la severidad de la afectación encontramos alteraciones de la formación del esmalte visibles como estrías, veteados, manchas marrones, 
etc. En el grado más severo la desmineralización es mucho más profunda pudiendo alcanzar la unión esmalte-dentina. Tras la erupción hay fracturas de la superficie y pronto se observan áreas opacas teñidas con aspecto de superficie descascarillada $(38,39)$.

El uso inapropiado de suplementos fluorados y el uso de fórmulas para infantes en la forma de concentrados en polvo han sido asociados a riesgo de fluorosis en áreas que cuentan con agua fluorada (1,38,44-47). Actualmente los suplementos fluorados están reconocidos por muchos autores como un factor de riesgo de fluorosis dental cuando se administran a niños menores de 5-6 años ya que la maduración preeruptiva de los dientes permanentes no se ha completado (34-36,48,49). Los factores metabólicos personales son determinantes (39) sin que podamos saber, a ciencia cierta, quien presenta una mayor predisposición por lo que las variables manejadas en la práctica son las dosis ingeridas y el estadio de maduración dentaria.

El nivel de ingesta de fluoruro por encima del cual se desarrollará fluorosis se estima en 0,05-0,07 mg F/kg peso corporal por día $(38,40,50)$. Ellwood et al.(51) proponen como dosis de riesgo leve $0,02 \mathrm{mg} / \mathrm{kg}$ por día y riesgo severo de fluorosis $0,1 \mathrm{mg} / \mathrm{kg}$ diarios.

\section{Preparados fluorados de uso tópico}

Buscando los efectos beneficiosos de los fluoruros e intentando minimizar los riesgos se acepta, actualmente, que la vía tópica es la más eficaz y segura para su administración.

Los preparados para administración tópica son los dentífricos, los enjuagues y colutorios, así como los barnices y geles para aplicación profesional con elevadas concentraciones de fluoruros (7,51-54).

\section{Los dentífricos fluorados en el niño}

Aunque la acción beneficiosa de los fluoruros procedentes de los dentífricos es indudable, la prescripción y utilización de los mismos debe hacerse teniendo en cuenta los posibles riesgos de intoxicación aguda o crónica (19,27,51,55-62).

Actualmente las sales fluoradas más utilizadas en los dentífricos son el fluoruro sódico y el monoflúorfosfato sódico. La eficacia de cada una de ellas ha sido muy estudiada y desde el punto de vista clínico no parece haber mucha diferencia en el comportamiento de una y otra formulación. La acción preventiva parece derivarse de la concentración de fluoruro presente (2,63-67). Jenkins (68) manifiesta que tras un cepillado con pasta que contenga 500, 1000 o 1500 ppm de fluoruro, seguido del enjuague con agua, las concentraciones de fluoruro la saliva están entre 60 y 250 ppm. Transcurridos 3 minutos la concentración baja a 3-11 ppm. A la media hora es 0,1-0,03 ppm y a la hora las concentraciones están ligeramente por encima de lo habitual (0,03-0,15 ppm).

Otros autores han estudiado las concentraciones de fluoruros en relación al tipo de enjuague con agua que se realice posteriormente. Un enjuague vigoroso disminuye la concentración de fluoruro de forma importante pero también aumenta el riesgo de ingestión $(64,69,70)$. Por el contrario el enjuagarse con un poco de agua durante largo rato puede tener un efecto beneficioso sobre las lesiones interproximales.

Los dentífricos de utilización más frecuente presentan una concentración de fluoruro de 500, 1000 o 1500 ppm (0,5; 1,0 y $1,5 \mathrm{mg}$ F/g de dentífrico). En los niños muy pequeños se ha propuesto la utilización de concentraciones menores de 500 ppm con el fin de disminuir el riesgo de fluorosis $(37,38,70)$. La evidencia científica todavía no es concluyente respecto a la menor acción protectora frente a la caries de estos dentífricos, a pesar de haberse establecido una relación directa entre concentración y efectividad $(1,37,70,71)$.

Los factores potenciales de riesgo de fluorosis asociados al uso de pastas dentales fluoradas por los niños pequeños incluyen la edad de inicio del cepillado, la frecuencia de cepillado, la concentración de fluoruro en el dentífrico, así como la cantidad de dentífrico aplicado al cepillo y luego deglutido $(1,38)$.

Con respecto a la edad de cepillado, el principal indicador es el uso de dentífrico fluorado antes de los 2 años (38). De otro lado, la ingestión de fluoruro de los dentífricos puede ser sustancial entre los niños pequeños, recordando que la capacidad de expectorar no está desarrollada hasta los 6-7 años $(61,62,70,72)$. Por último, a pesar de las recomendaciones sobre la cantidad de dentífrico que deben utilizar los niños durante el cepillado, el equivalente al “tamaño de un guisante” o 0,25 g (58,59), en la práctica los niños utilizan una cantidad mucho mayor de dentífrico (55).

Levy et al. (61) estudiando la ingestión de dentífrico en niños entre 40 y 48 meses, manifiestan que la ingestión es de hasta el $62 \%$ estando significativamente asociada a la cantidad de dentífrico usada. Naccache et al. (62), en una muestra de niños de 2 a 7 años, hallaron que la cantidad promedio de pasta usada es $0,5 \mathrm{~g}$ en cada cepillado y obtienen una ingestión del $65 \%$ en los niños de dos años, $49 \%$ a los 3-4 años y 34\% a los 6-7 años. Cochran et al. (37), en una muestra proveniente de siete ciudades europeas, determinaron que la cantidad de pasta dental utilizada por los niños de 1,5-2,5 años promedia los 0,36 g. 0,41 g para los niños de 2.5-3.5 años y 0,49 g para los niños mayores de 3.5 años. La proporción de fluoruro ingerido fue $64 \%$. Los niños de menor edad ingirieron entre 80\% y $100 \%$ del fluoruro dispensado y los niños mayores de 3.5 años ingirieron una media de $53 \%$.

Cabe destacar que los dentífricos comercializados como infantiles, no necesariamente contienen una baja concentración de fluoruro, sino distinto sabor y presentación. Se ha demostrado que son usadas y, por tanto, ingeridas, mayores cantidades de dentífrico saborizado por los niños en comparación con las cantidades de dentífrico para adultos $(37,66,70)$. Otros autores, al comparar la utilización de pasta familiar (1450 ppm) y pasta infantil (400 ppm) por niños de treinta meses de edad, no encuentran diferencias significativas en la cantidad utilizada para un cepillado con cada una de las concentraciones $(0,37 \mathrm{~g}$ con la pasta familiar y $0,34 \mathrm{~g}$ con la pasta infantil), ni en el porcentaje de pasta ingerida (77\% con la pasta familiar y $69 \%$ con la pasta infantil). La cantidad de fluoruro ingerido oscila entre 0,1 mg para los niños que utilizan pasta infantil y 0,42 mg para los que se cepillan con la familiar (73)

La contribución promedio de la pasta dental a la ingesta diaria total de fluoruro en niños de 1.5-2.5 años de edad se ubica en el rango de 43-71\% (50). 


\section{Riesgo de toxicidad de los dentífricos}

En base a estos y otros estudios, puede deducirse que el riesgo de toxicidad crónica e incluso aguda por ingestión excesiva de pasta dental es, en el caso de los niños, real.

En la Tabla 1 se han expresado los gramos de dentífrico que necesitaría ingerir un niño de 12,5 kg (aproximadamente 2 años de edad) y de $20 \mathrm{~kg}$ (aproximadamente 5-6 años) para que la dosis tuviera riesgo de toxicidad aguda. Considerando que el contenido de los tubos por lo general es de $75-100 \mathrm{ml}$, podemos observar que en los tubos de pasta dental de concentraciones de 1500 ppm y 1000 ppm hay cantidad suficiente para un accidente agudo en el caso que el niño la ingiera (aproximadamente medio tubo por los niños de 2 años y 1 tubo por los niños de 5-6 años). Esto nos debería hacer reflexionar y considerar a las pastas dentífricas como un medicamento que no debe estar al alcance de los niños.
Del mismo modo, en la Tabla 2 se ha estimado la cantidad de dentífrico necesario para que se derive un riesgo severo o leve de fluorosis dental por la ingestión continuada de parte del dentífrico durante el cepillado. Si asumimos que el cepillado se realiza 2 veces al día, cada vez con un promedio de 0,5 gr de dentífrico (62), y que el niño preescolar traga el 50\% ó más $(73,62)$, las posibilidades de producirse fluorosis son reales en estos niños en los que las coronas dentarias se están formando, especialmente si utilizan los dentífricos con alta concentración de fluoruro. A partir de los 5-7 años la ingesta de fluoruros tiene una repercusión menor en ese aspecto.

\section{Enjuagues fluorados}

La utilización de enjuagues fluorados tras el cepillado dental es una práctica cada vez más extendida y de comprobados efectos anticaries $(53,54,74)$

Estos preparados tienen un efecto

Tabla 1. Cantidad de dentífrico con riesgo de toxicidad aguda en relación a la concentración de fluoruro y peso del niño.

\begin{tabular}{lcc}
\hline Concentración de fluoruro & $12,5 \mathrm{~kg}$ (»2 años) & $20 \mathrm{~kg}$ (»5-6 años) \\
\hline $1500 \mathrm{ppm}(1,5 \mathrm{mg} / \mathrm{g})$ & $42 \mathrm{~g}$ dentífrico & 66 g dentífrico \\
$1000 \mathrm{ppm}(1,0 \mathrm{mg} / \mathrm{g})$ & $62,5 \mathrm{~g}$ dentífrico & 100 g dentífrico \\
$500 \mathrm{ppm}(0,5 \mathrm{mg} / \mathrm{g})$ & $125 \mathrm{~g}$ dentífrico & 200 g dentífrico \\
$400 \mathrm{ppm}(0,4 \mathrm{mg} / \mathrm{g})$ & $156 \mathrm{~g}$ dentífrico & 250 g dentífrico \\
\hline
\end{tabular}

Dosis Tóxica Probable (DTP) : $5 \mathrm{mg} / \mathrm{kg}$

Tabla 2. Cantidad de dentífrico que determina riesgo de fluorosis dental por ingestión diaria del $50 \%$ de dentífrico cepillándose 2 veces/día.

\begin{tabular}{|c|c|c|c|c|}
\hline \multirow[b]{2}{*}{$\begin{array}{l}\text { Concentración } \\
\text { de fluoruro }\end{array}$} & \multicolumn{2}{|c|}{ 12,5 kg (»2 años) } & \multicolumn{2}{|c|}{20 kg (»5-6 años) } \\
\hline & $\begin{array}{l}\text { Riesgo severo } \\
\text { de fluorosis }\end{array}$ & $\begin{array}{l}\text { Riesgo leve } \\
\text { de fluorosis }\end{array}$ & $\begin{array}{l}\text { Riesgo severo } \\
\text { de fluorosis }\end{array}$ & $\begin{array}{l}\text { Riesgo leve } \\
\text { de fluorosis }\end{array}$ \\
\hline 1500 ppm (1,5 mg/g) & $0,83 \mathrm{~g} / \mathrm{Dt}$ & $0,17 \mathrm{~g} / \mathrm{Dt}$ & $1,33 \mathrm{~g} / \mathrm{Dt}$ & $0,27 \mathrm{~g} / \mathrm{Dt}$ \\
\hline 1000 ppm (1 mg/g) & $1,25 \mathrm{~g} / \mathrm{Dt}$ & $0,25 \mathrm{~g} / \mathrm{Dt}$ & $2 \mathrm{~g} / \mathrm{Dt}$ & $0,4 \mathrm{~g} / \mathrm{Dt}$ \\
\hline 500 ppm (0,5 mg/g) & $2,50 \mathrm{~g} / \mathrm{Dt}$ & $0,5 \mathrm{~g} / \mathrm{Dt}$ & $4 \mathrm{~g} / \mathrm{Dt}$ & $0,8 \mathrm{~g} / \mathrm{Dt}$ \\
\hline 400 ppm (0,4 mg/g) & $3,12 \mathrm{~g} / \mathrm{Dt}$ & $0,62 \mathrm{~g} / \mathrm{Dt}$ & $5 \mathrm{~g} / \mathrm{Dt}$ & $1 \mathrm{~g} / \mathrm{Dt}$ \\
\hline
\end{tabular}

Dosis de riesgo severo de fluorosis: $0,1 \mathrm{mg} \mathrm{F}^{-} / \mathrm{kg}$

Dosis de riesgo leve de fluorosis: $0,02 \mathrm{mg} \mathrm{F}^{-3} / \mathrm{kg}$

Dt: Dentífrico

Tabla 3. Cantidad de enjuague con riesgo de toxicidad aguda en relación a la concentración de fluoruro y peso del niño.

\begin{tabular}{llll}
\hline Enjuague & Cantidad fluoruro & $12,5 \mathrm{~kg}$ (»2 años) & $20 \mathrm{~kg}$ (»5-6 años) \\
FNa 0,05\% & $0,23 \mathrm{mg} / \mathrm{ml}$ & $272 \mathrm{ml}$ & $434 \mathrm{ml}$ \\
FNa 0,2\% & $0,91 \mathrm{mg} / \mathrm{ml}$ & $68 \mathrm{ml}$ & $110 \mathrm{ml}$ \\
\hline
\end{tabular}

Dosis Tóxica Probable (DTP): 5 mg/kg

cariostático. Sin embargo, como todos los medicamentos, tienen efectos adversos y posibles riesgos que se derivan, generalmente, de la utilización de productos inadecuados o dosis incorrectas $(74,75)$.

Es fundamental valorar la capacidad del niño de expectorar adecuadamente pero esto no ocurre antes de los 6-7 años de edad, de modo que la utilización de estos preparados antes de esta edad tiene un serio riesgo de que sean ingeridos en parte y sus efectos se sumarán a la cantidad de fluoruro ingerido procedente de la pasta dental fluorada, si también la usa.

Se utilizan preparados de fluoruro sódico $(\mathrm{NaF})$ al 0,20\% (910 ppm F-) para uso semanal y de $0,05 \%$ (230 ppm F-) para uso diario. El tiempo de enjuague recomendado es de 1 minuto, con una cantidad de 5-10 $\mathrm{ml}$. El uso de $5 \mathrm{ml}$ de la solución de uso diario puede ser más conveniente en niños escolares debido al riesgo de ingestión $(76,77)$. Es muy importante que se utilicen únicamente preparados libres de alcohol. Los enjuagues comercializados para ser utilizados por niños no suelen contenerlo en su composición.

Los riesgos del uso de enjuagatorios fluorados se relacionan principalmente a la toxicidad aguda y crónica por ingestión de fluoruro y a la toxicidad aguda por ingestión de etanol. La ingestión de enjuagues que contengan alcohol puede llegar a causar la muerte por toxicidad aguda (78). Sin llegar a esto, es frecuente que el etanol tenga un efecto irritante local con manifestaciones como descamación, ulceraciones de la mucosa, gingivitis, petequias o cuadros alérgicos. Consideración especial merece la posible relación entre el cáncer oral y la utilización repetida de productos locales que contengan alcohol (75).

Incluso utilizando preparados libres de alcohol hay riesgo de toxicidad aguda si el niño bebe la cantidad suficiente (74). En la Tabla 3 se expresa la cantidad de enjuague que podría inducir toxicidad aguda al ser ingerido por un niño. Aunque los datos indican la seguridad del uso de las formulaciones de $\mathrm{NaF}$ $0.05 \%$, es importante observar que la DTP estaría disponible en una sola botella (presentación de $120 \mathrm{ml}$ ) para las concentraciones de $0,2 \% \mathrm{NaF}$. 
Tabla 4. Cantidad de fluoruro ingerido según la concentración y cantidad de preparado.

\begin{tabular}{lcll}
\hline Enjuague & Cantidad & Cantidad de fluoruro & 25\% de ingestión \\
\hline FNa 0,05\% & $10 \mathrm{ml} /$ día & $2,3 \mathrm{mg} /$ día & $0,57 \mathrm{mg} /$ día \\
(diario) & $5 \mathrm{ml} /$ día & $1,15 \mathrm{mg} /$ día & $0,29 \mathrm{mg} /$ día \\
FNa 0,2\% & $10 \mathrm{ml} / \mathrm{sem}$. & $9,1 \mathrm{mg} /$ día & $2,27 \mathrm{mg} / \mathrm{sem}$. \\
(semanal) & $5 \mathrm{ml} / \mathrm{sem}$. & $4,55 \mathrm{mg} /$ día & $1,13 \mathrm{mg} / \mathrm{sem}$. \\
\hline
\end{tabular}

Dosis de riesgo severo de fluorosis: $0,1 \mathrm{mg}$ F-kg

Dosis de riesgo leve de fluorosis: 0,02 mg F -/kg

Los niños menores de 6 años tragan una cantidad considerable del líquido depositado en su boca. Diversos estudios $(26,54,74)$ sitúan esta cantidad entre el 22$40 \%$. En la Tabla 4 se expresa, en mg, el fluoruro ingerid o durante los enjuagues con preparados de distintas concentraciones. Puede deducirse que, en los niños pequeños, la ingestión continuada supone un riesgo real de fluorosis.

\section{Preparados para uso profesional}

Para la prevención de la caries dental en el niño se utilizan, rutinariamente en el consultorio, productos con alta concentración de fluoruro, en la forma de geles o barnices.

El gel más usado para aplicación profesional es el flúorfosfato acidulado (FPA) al 1,23\%, es decir, en concentración de 12,300 ppm o 12,3 mg/ml de fluoruro (79). $\mathrm{Al}$ entrar en contacto con el diente, se disuelve un espesor mínimo de esmalte liberando calcio que con el fluoruro del gel forma fluoruro cálcico amorfo que vuelve a precipitar haciéndolo más resistente a las caídas del pH (80).

Aproximadamente se emplean $5 \mathrm{ml}$ de gel en una cubeta, representando una exposición potencial a 61,5 mg de ión fluoruro. Para que ocurra un accidente agudo sería necesario que un niño de $12,5 \mathrm{~kg}$ ingiera $5 \mathrm{ml} \mathrm{y} 8,1 \mathrm{ml}$ uno de $20 \mathrm{~kg}$ (79). El sabor y la consistencia de la preparación hacen muy difícil que esto ocurra, pero es obligado aspirar la saliva durante la aplicación pues si se ingiere en exceso puede provocar molestias gástricas. Debido al riesgo de sobreingestión, el uso de geles en niños pequeños no está recomendado. Teniendo en cuenta estas consideraciones, y puesto que el preparado se aplica bajo supervisión a intervalos de 4-6 meses, no existe riesgo de fluorosis (54).

Los barnices fluorados de uso más difundido contienen en su formulación fluoruro de sodio (NaF) al 5\%, equivalente a $22,6 \mathrm{mg} / \mathrm{ml}$ o 22,600 ppm F (81). Se aplican sobre los dientes en muy peque- ña cantidad, en promedio 0,3-0,5 ml, por lo que constituyen una alternativa segura frente al uso de los geles. Para que se produzca un accidente agudo sería necesaria la ingestión de 2,5 ml en un niño de 10 kg ó 5 ml en un niño de $20 \mathrm{~kg}$.

Los barnices tienen una eficacia demostrada, una técnica de aplicación muy fácil que los hacen muy útiles en los pacientes jóvenes y prácticamente no se describen complicaciones $(81,82)$.

\section{Conclusiones}

1 Los médicos pediatras, médicos de familia y odontopediatras son responsables de un uso racional del flúor como elemento de eficacia demostrada en la prevención de la caries dental. Debe ser considerado un medicamento y, por tanto, él será quien decida la composición, vía de administración, dosificación y pautas generales acordes con el grado de salud o enfermedad de su boca, la edad y el estadio del desarrollo dentario.

2 Es fundamental estimar en cada paciente el Aporte Total de Flúor a partir de las concentraciones de flúor contenido en las aguas de bebida; de otras fuentes alimentarias como la sal, el té o la leche; de la ingestión de suplementos fluorados y de los hábitos de higiene bucal valorando en éstos la concentración de flúor presente en la pasta utilizada, las veces que se cepilla el día y la utilización de enjuagues fluorados.

3 Deberán prescribirse pastas dentales que no superen 1000 ppm insistiendo en que la cantidad sea muy pequeña (similar a un guisante), dos veces por día. Los niños menores de 3 años deben cepillarse sin pasta y entre los 3 y 6 años deberá vigilarse la cantidad para disminuir el riesgo de fluorosis.

4 Antes de los 6-7 años el niño no puede realizar enjuagues sin tragar una parte significativa del producto. Los colutorios no deberían prescribirse antes de esta edad y solamente cuando se haya probado que el niño es capaz de expectorar adecuadamente podrán incorporarse a las medidas preventivas de salud bucal.

5 Se prescribirán, únicamente, colutorios libres de alcohol. Los preparados infantiles que contienen fluoruro al 0,5\% para uso diario suelen estar exentos pero es necesario comprobarlo en cada marca comercial.

6 Los padres deben seguir las instrucciones que se les ha dado en cuanto a la composición de los medicamentos, cantidades y formas de usarlos. Deben aprender que no son productos cosméticos sino de tratamiento y, por tanto, dejarlos fuera del alcance de los niños e ir entrenando a éstos, según van creciendo, en el uso racional de los mismos.

7 Es necesario concienciar a los fabricantes para que las preparaciones tengan una composición y una forma de presentación que facilite la labor de los profesionales y los padres. Las instrucciones en el envase del producto deben estar claras, el tamaño de las presentaciones no debe ser suficiente para provocar accidentes agudos y las boquillas serán pequeñas para facilitar el dispensado de cantidades mínimas.

\section{Referencias bibliográficas}

1. Whelton HP, Ketley CE, McSweeney F, O'Mullane DM. A review of fluorosis in the European Union: prevalence, risk factors and aesthetic issues. Community Dent Oral Epidemiol 2004;32(Suppl 1):9-18.

2. O'Mullane DM. Introduction and rationale for the use of fluoride for caries prevention. Int Dent J 1994;44(3 Suppl 1):257-61.

3. Sköld UM, Lindvall AM, Rasmusson CG, Birkhed D, Klock B. Caries incidence in adolescents with low caries prevalence after cessation of weekly fluoride rinsing. Acta Odontol Scand 2001;59:69-73.

4. Maupome G, Clark DC, Levy SM, Berkowitz J. Patterns of dental caries following the cessation of water fluoridation. Community Dent Oral Epidemiol 2001;29:37-47.

5. Seppä L, Kärkkäinen S, Hause H. Caries trends 1992-1998 in two lowfluoride Finnish towns formerly with and without fluoridation. Caries Res 2000;34:462-8. 
6. US Public Health Service, Centers for Disease Control and Prevention: Recommendations for using fluoride to prevent and control dental caries in the United States. MMWR Morb Mortal Wkly Rep 2001;50(RR14):1-42.

7. Clarkson JJ, McLoughlin J. Role of fluoride in oral health promotion. Int Dent J 2000;50:119-28.

8. Künzel W, Fischer T, Lorenz R, Brühmann $S$. Decline of caries prevalence after the cessation of water fluoridation in the former East Germany. Community Dent Oral Epidemiol 2000;28:382-9.

9. Limeback H. A re-examination of the pre-eruptive and post-eruptive mechanism of the anti-caries effects of fluoride: is there any anti-caries benefit from swallowing fluoride? Community Dent Oral Epidemiol 1999;27:62-71.

10. Featherstone JD. Prevention and reversal of dental caries: role of low fluoride. Community Dent Oral Epidemiol 1999;27:31-40.

11. Larse MJ, Richards A. The influence of saliva on the formation of calcium fluoride-like material on human dental enamel. Caries Res 2001;35:57-60.

12. Takagi S, Liao H, Chow LC. Effect of tooth-bound fluoride on enamel demineralization/remineralization in vitro. Caries Res 2000;34:281-8.

13. Ramos Atance JA. El flúor y su papel en la remineralización. En: Bioquímica bucodental. Madrid: Ed Síntesis S.A. 1996;105-114.

14. Moreno EC. Role of Ca-P-F in caries prevention: chemical aspects. Int Dent J 1993;43:71-80.

15. ten Cate JM. Current concepts on the theories of the mechanism of action of fluoride. Acta Odontol Scand 1999;57:325-9.

16. Ogaard B. The cariostatic mechanism of fluoride. Compend Contin Educ Dent 1999;20(1 Suppl):10-7.

17. Al-Khateeb S, Exterkate R, AngmarMänson B. Effect of acid-etching on remineralization of enamel white spot lesions. Acta Odontol Scand 2000;58:31-6.

18. Modesto A, Costa Lima K, de Uzeda M. Effects of three different infant dentifrices on biofilms and oral microorganisms. J Clin Pediatr Dent 2000;24:237-43.

19. Modesto A, Lima KC, de Uzeda M. Effects of solutions used in infants' oral hygiene on biofilms and oral microorganisms. ASDC J Dent Child 2000;67:338-44.

20. Estupiñán-Day SR, Baez R, Horowitz H, Warpeha R, Sutherland B, Thamer M. Salt fluoridation and dental caries in Jamaica. Community Dent Oral Epidemiol 2001;29:247-52.

21. Ketley CE, Lennon MA. Determination of fluoride intake from urinary fluoride excretion data in children drinking fluoridated school milk. Caries Res 2001;35:252-7.

22. Boros I, Keszler P, Bánóczy J. Fluoride concentrations of unstimulated whole and labial gland saliva in young adults after fluoride intake with milk. Caries Res 2001;35:167-72.

23. Simpson A, Shaw L, Smith AJ. The bio-availability of fluoride from black tea. J Dent 2001;29:15-21.

24. Warren JJ, Levy SM. Systemic fluoride. Sources, amounts, and effects of ingestion. Dent Clin North Am 1999;43:695-711.

25. Fomon SJ, Ekstrand J. Fluoride Intake by Infants. J Public Health Dent 1999;59:229-34.

26. Levy SM, Guha-Chowdhury N. Total fluoride intake and implication for dietary fluoride supplementation. J Public Health Dent 1999;59:211-23.

27. Guha-Chowdhury N, Drummond BK, Smillie AC. Total fluoride intake in children aged 3 to 4 years-A longitudinal study. J Dent Res 1996;75:1451-7.

28. Ekstrand J, Spak CJ, Vogel G. Pharmacokinetics of fluoride in man and its clinical relevance. J Dent Res 1990;69 Spec No:550-5.

29. Whitford GM. Fluoride metabolism and excretion in children. J Public Health Dent 1999;59:224-8.

30. Whitford GM. The physiological and toxicological characteristics of fluoride. J Dent Res 1990;69:539-49.

31. Ekstrand J, Oliveby A. Fluoride in the oral environment. Acta Odontol Scand 1999; 57:330-3.

32. Whitford GM. Acute and chronic fluoride toxicity. J Dent Res 1992;71:1249-54.

33. Shulman JD, Wells LM. Acute fluoride toxicity from ingesting home-use dental products in children, birth to 6 years of age. J Public Health Dent 1997;57:150-8.

34. Newbrun E. The case for reducing the current council on dental therapeutics fluoride supplementation schedule. J Public Health Dent
1999;59:263-8.

35. Ismail Al, Bandekar RR. Fluoride supplements and fluorosis: a metaanalysis. Community Dent Oral Epidemiol 1999;27:48-56.

36. Riordan PJ. Fluoride supplements for young children: an analysis of the literature focusing on benefits and risks. Community Dent Oral Epidemiol 1999;27:72-83.

37. Cochran JA, Ketley CE, Duckworth RM, et al. Development of a standardized method for comparing fluoride ingested from toothpaste by 1.53.5-year-old children in seven European countries. Part 1: Field work. Community Dent Oral Epidemiol 2004;32 (Suppl 1):39-46.

38. Mascarenhas AK. Risk factors for dental fluorosis: A review of the recent literature. Pediatr Dent 2000;22:269-77.

39. DenBesten PK. Biological mechanisms of dental fluorosis relevant to the use fluoride supplements. Community Dent Oral Epidemiol 1999;27:41-7.

40. van Loveren C, Ketley CE, Cochran JA, Dochworth RM, O’Mullane DM. Fluoride ingestion from toothpaste: fluoride recovered from the toothbrush, the expectorate and the afterbrush rinses. Community Dent Oral Epidemiol 2004;32(Suppl 1): 54-61.

41. O’Mullane DM, Ketley CE, Cochran JA, et al. Fluoride ingestion from toothpaste: conclusions of European Union-funded multicentre project. Community Dent Oral Epidemiol 2004;32(Suppl 1): 74-76.

42. Warren JJ, Levy SM, Kanellis MJ. Prevalence of dental fluorosis in the primary dentition. J Public Health Dent 2001;61:87-91.

43. Levy SM, Hillis SL, Warren JJ, et al. Primary tooth fluorosis and fluoride intake during the first year of life. Community Dent Oral Epidemiol 2002;30:286-95.

44. Fomon SJ, Ekstrand J, Ziegler EE. Fluoride intake and prevalence of dental fluorosis: Trends in fluoride intake with special attention to infants. J Public Health Dent 2000;60:131-9.

45. Buzalaf MAR, Damante CA, Trevizani LMM, Granjeiro JM. Risk of fluorosis associated with infant formulas prepared with bottled water. ASDC J Dent Child 2004;71:110-13. 46. Anderson WA, Pratt I, Ryan MR, 
Flynn A. A probabilistic estimation of fluoride intake by infants up to the age of 4 months from infant formula reconstituted with tap water in the fluoridated regions of Ireland. Caries Res 2004;38:421-9.

47. Buzalaf MA, Granjeiro JM, Damante CA, de Ornelas F. Fluoride content of infant formulas prepared with deionized, bottled mineral and fluoridated drinking water. ASDC J Den Child 2000; 68:37-41.

48. Adair SM. Overview of the history and current status of fluoride supplementation schedules. J Public Health Dent 1999;59:252-8.

49. Horowitz HS. The role of dietary fluoride supplements in caries prevention. J Public Health Dent 1999;59:205-10.

50. Cochran JA, Ketley CE, Duckworth RM, et al. Development of a standardized method for comparing fluoride ingested from toothpaste by 1.53.5-year-old children in seven European countries. Part 2: Ingestion results. Community Dent Oral Epidemiol 2004;32 (Suppl 1): 47-53.

51. Ellwood RP, Blinkhorn AS, Davies RM. Fluoride: How to maximize the benefits and minimize the risks. Dent Update 1998;25:365-72.

52. Stamm JW. The value of dentifrices and mouthrinses in caries prevention. Int Dent J 1993; 43(6 Suppl 1):517-27.

53. Ripa LW. A critique of topical fluoride methods (dentifrices, mouthrinses, operator-, and self-applied gels) in an era of decreased caries and increased fluorosis prevalence. J Public Health Dent 1991;51:23-38.

54. Sttokey GK. Rewiew of fluorosis risk of self-applied topical fluorides: dentifrices mouthrinses and gels. Community Dent Oral Epidemiol 1994; 22:181-6.

55. Pang DTY, Vann WF. The use of fluoride-containing toothpastes in young children: the scientific evidence for recommending a small quantity. Pediatr Dent 1992;14:384-7.

56. Pereira AC, Da Cunha FL, Meneghim MdeC, Werner CW. Dental caries and fluorosis prevalence study in a nonfluoridated Brazilian community: Trend analysis and toothpaste association. ASDC J Dent Child 2000; 67:132-5.

57. Chan JC, O’Donnell D. Ingestion of fluoride dentifrice by a group of mentally handicapped children during tootbrushing. Quintessence Int 1996;27:409-11.

58. DenBesten P, Ko HS. Fluoride levels in whole saliva of preschool children after brushing with $0.25 \mathrm{~g}$ (peasized) as compared to $1.0 \mathrm{~g}$ (fullbrush) of a fluoride dentifrice. Pediatr Dent 1996;18:277-80.

59. Villena RS. An investigation of the transverse technique of dentifrice application to reduce the amount of fluoride dentifrice for young children. Pediatr Dent 2000; 22:312-7.

60. Levy SM, Kiritsy MC, Slager SL, Warren JJ, Kohout FJ. Patterns of fluoride dentifrice use among infants. Pediatr Dent 1997; 19:50-55.

61. Levy SM, McGrady JA, Bhuridej P, Warren JJ, Heilman JR, Wefel JS. Factors affecting dentifrice use and ingestion among a sample of U.S. preschoolers.Pediatr Dent 2000; 22:389-94.

62. Naccache H, Simard PL, Trahan L, Brodeur JM, Demers M, Lachapelle D. Factors affecting the ingestion of fluoride dentifrice by children. J Public Health Dent 1992;52:222-6.

63. Itthagarun A, Wei SH, Wefel JS. The effect of different commercial dentifrices on enamel lesion progression: an in vitro $\mathrm{pH}$-cycling study. Int Dent J 2000;50:21-8.

64. Stephen KW. Dentifrices: recent clinical findings and implication for use. Int Dent J 2000;43:549-53.

65. Biesbrock AR, Gerlach RW, Bollmer BW, Faller RV, Jacobs SA, Bartizek RD. Relative anti-caries efficacy of $1100,1700,2200$, and 2800 ppm fluoride ion in a sodium fluoride dentifrice over 1 year. Community Dent Oral Epidemiol 2001;29:382-9.

66. Levy SM, Maurice TJ, Jakobsen JR. A pilot study of preschoolers'use of regular-flavored dentifrices and those flavored for children. Pediatr Dent 1992;14:388-91.

67. Rock WP. Young children and fluoride toothpaste. Br Dent J 1994; 177:17-20.

68. Jenkins GN. Review of fluoride research since 1959. Arch Oral Biol 1999; 44:985-92.

69. Sjögren K, Ekstrand J, Birkhed D. Effect of water rinsing after toothbrushing on fluoride ingestion and absorption. Caries Res
1994;28:455-59

70. Warren JJ, Levy SM. Areview of fluoride dentifrice related to dental fluorosis. Pediatr Dent 1999;21:266-72.

71. Ammari AB, Bloch-Zupan A, Ashley PF. Systematic review of studies comparing the anti-caries efficacy of children's toothpaste containing 600 ppm of fluoride or less with high fluoride toothpastes of $1,000 \mathrm{ppm}$ or above. Caries Res 2003;37:85-92.

72. Ketley CE, Cochran JA, Holbrook WP, et al. Urinary fluoride excretion by preschool children in six European countries. Community Dent Oral Epidemiol 2004; 32 (Suppl 1): 62-68.

73. Bentley EM, Ellwood RP, Davies RM. Fluoride ingestion from toothpaste by young children. $\mathrm{Br}$ Dent J 1999;186:460-2.

74. Adair SM. The role of fluoride mouthrinses in the control of dental caries: a brief review. Pediatr Dent 1998; 20:101-4.

75. Gagari E, Kabani S. Adverse effects of mouthwash use. Oral Surg Oral Med Oral Pathol Oral Radiol Endod 1995;80:432-9.

76. FDI Commission. Mouthrinses and dental caries. Int Dent J 2002;52:33745.

77. Inaba D, Kawasaki K, Iijima Y, et al. Enamel fluoride uptake from mouthrinse solutions with different $\mathrm{NaF}$ concentrations. Community Dent Oral Epidemiol 2002;30:248-53.

78. Shulman JD, Wells LM. Acute ethanol toxicity from ingesting mouthwash in children younger than 6 years of age. Pediatr Dent 1997;19:405-8.

79. Marinho VCC, Higgins JPT, Logan $\mathrm{S}$, Sheiham A. Systematic review of controlled trials on the effectiveness of fluoride gels for the prevention of dental caries in children. J Dent Educ 2003;67:448-58.

80. Ogaard B. CaF(2) formation: cariostatic properties and factors of enhancing the effect. Caries Res 2001;35(Suppl 1):40-4.

81. Beltrán-Aguilar DE, Goldstein JW, Lockwood SA. Fluoride varnishes: A review of their clinical use, cariostatic mechanism, efficacy and safety. JADA 2000;131:589-96.

82. Castillo L. Importancia clínica de los barnices fluorados. Bol Asoc Odontol Arg para Niños 2001;30(1):19-23. 